\title{
Idiopathic Pulmonary Fibrosis: What Is The Best Treatment?
}

Xiaoping Wu, MD', Robert J. Kaner, MD ${ }^{1,2}$, and Fernando J. Martínez, MD, MS ${ }^{1,2}$

${ }^{1}$ Division of Pulmonary and Critical Care Medicine, and ${ }^{2}$ Department of Genetic Medicine, Weill Cornell Medicine, New York, NY, USA

\section{ABSTRACT}

Idiopathic pulmonary fibrosis (IPF) is a disease of chronic progressive interstitial pneumonia limited to the lungs. There is growing interest in this disorder as its incidence has increased over time in most countries around the world. This is likely related to an aging population, increased awareness of the disease, and increasingly sensitive imaging technology. Considerable energy has been devoted to creating an improved understanding of its pathogenesis and developing novel therapies. Although dozens of drugs have been studied for the treatment of IPF, only two, pirfenidone and nintedanib, are currently recommended by international guidelines to slow the disease progression. We review the drugs that have been evaluated as IPF therapy over the past three decades, including the currently recommended pirfenidone and nintedanib, note ongoing clinical trials and provide insights into future directions. (BRN Rev. 2017;3:86-101)

Corresponding author: Fernando J. Martínez, fjm2003@med.cornell.edu

Key words: Combination therapy. Idiopathic pulmonary fibrosis. Nintedanib. Pirfenidone. Precision medicine. 


\section{INTRODUCTION}

Idiopathic pulmonary fibrosis (IPF) is a disease of chronic progressive interstitial pneumonia limited to the lungs ${ }^{1}$. There is growing interest in IPF as its incidence has increased over time in most countries around the world. This is likely related to our aging population, increased awareness of the disease among physicians, and increasingly sensitive imaging technology. A recent epidemiology study estimates an incidence range of 2.8-9.3 per 100,000 persons per year when limited to data after the year 2000 using narrow criteria for diagnosis in Europe and North America ${ }^{2}$. Considerable energy has been apportioned to understanding the pathogenesis of IPF and developing novel therapies in the past few decades. Dozens of drugs have been studied for the treatment of IPF but only two, pirfenidone and nintedanib, are currently recommended by international guidelines ${ }^{3}$ to slow the progression of disease. Here, we review the drugs that have been evaluated as treatments for IPF over the past three decades, including the currently recommended pirfenidone and nintedanib, as well as ongoing clinical trials and future directions.

\section{PREVIOUS ERA}

\section{Prednisone and immunosuppressive agents}

Prior to 2012, the conventional treatment of IPF involved glucocorticoids in combination with either azathioprine or cyclophosphamide as recommended by the American Thoracic Society (ATS) and European Respiratory Society (ERS) ${ }^{4}$ based on a small case series and small prospective trials ${ }^{5-7}$. It is important to note that these studies were performed prior to the recognition of nonspecific interstitial pneumonia (NSIP) as a distinct, treatment-responsive entity and likely included patients with this histopathologic pattern, who are excluded from more recent IPF trials.

The landmark PANTHER-IPF (Prednisone, Azathioprine, and N-Acetylcysteine: A Study That Evaluates Response in Idiopathic Pulmonary Fibrosis) trial evaluated prednisone/ azathioprine/N-acetylcysteine (NAC) compared to NAC alone and placebo. The triple therapy arm of the study was prematurely stopped after interim analysis demonstrated that the triple drug regimen was associated with increased mortality, hospitalizations, and serious adverse events without improvement in lung function in the survivors when compared with placebo ${ }^{8}$.

\section{$\mathrm{N}$-acetylcysteine}

The NAC monotherapy versus placebo arm of the PANTHER trial proceeded to completion and demonstrated no difference in change in forced vital capacity (FVC) at 60 weeks 9 . A subsequent randomized trial of NAC versus placebo on a background of pirfenidone suggested a possible deleterious effect of the combination on lung function decline and the occurrence of photosensitivity ${ }^{10}$. Interestingly, a post hoc analysis of PANTHER participants suggested that patients with a TT genotype in the toll-interacting protein (TOLLIP) single nucleotide polymorphism (SNP) had a significant reduction in the composite endpoint (death, lung transplantation, hospitalization, or $\geq 10 \%$ decline in forced vital 
capacity [FVC]) in response to NAC therapy but those with CC genotype had a non-significant trend toward harm ${ }^{11}$.

\section{Immunomodulators}

A preliminary study of interferon $\beta-1 \alpha$ failed to show any significant benefit on pulmonary function, oxygenation, or disease progression in $\mathrm{IPF}^{12}$. However, it demonstrated that large, multi-centre, placebo-controlled trials in IPF were feasible.

Investigators went on to conduct a small study of subcutaneous interferon gamma therapy in IPF patients that resulted in lung function benefits ${ }^{13}$. A large, multinational, randomized controlled study unfortunately did not show any benefit ${ }^{14}$. A potential trend towards improved survival in patients who were treated with interferon gamma-1b prompted a larger, randomized placebo-controlled trial (INSPIRE) to evaluate whether the drug could improve survival in IPF patients with mild to moderate physiological impairment. The study was terminated early due to futility after the second interim analysis ${ }^{15}$.

IPF patients have elevated tumor necrosis factor alpha (TNF- $\alpha$ ) messenger ribonucleic acid (mRNA) levels in their alveolar epithelial cells and macrophages ${ }^{16}$. In a murine model of pulmonary fibrosis, a TNF- $\alpha$ antagonist attenuated lung collagen deposition after injury with bleomycin or silica ${ }^{17}$. A subsequent randomized, placebo-controlled trial of etanercept, a fusion protein which binds to TNF- $\alpha$, was well tolerated but demonstrated no significant effect on pulmonary physiology at 48 weeks $^{18}$. A similar trial involving imatinib, an intracellular tyrosine kinase inhibitor that inhibits downstream signalling from platelet derived growth factor (PDGF) and transforming growth factor-beta (TGF- $\beta$ ) receptors, yielded disappointing results with no effect on disease progression or survival at 96 weeks and an increased risk of adverse events ${ }^{19}$.

\section{Endothelin receptor antagonists}

IPF patients also have increased endothelin-1 expression in airway epithelial cells and type II pneumocytes ${ }^{20}$. Bosentan, an endothelin receptor antagonist, was studied in the Bosentan Use in Interstitial Lung Disease (BUILD)-1 and BUILD-3 trials which found no difference in mortality, disease progression, FVC, or health-related quality of life ${ }^{21-23}$. Macitentan similarly did not appear to significantly improve these outcomes in IPF patients in the MUSIC trial ${ }^{24}$. Despite experimental lung fibrosis animal models suggesting a potential benefit of endothelin type A receptor antagonists, ARTEMIS-IPF demonstrated a higher likelihood of harm from ambrisentan therapy ${ }^{25}$.

\section{Anticoagulation}

A small and methodologically flawed study of warfarin plus prednisolone versus prednisolone alone suggested a reduction in mortality associated with IPF acute exacerbation ${ }^{26}$. Warfarin was compared to placebo in a rigorous study by Idiopathic Pulmonary Fibrosis Clinical Research Network (IPFnet) investigators. Importantly, this trial was terminated early after interim analysis showed a significant increase in all-cause mortality not associated with bleeding complication and increase in combined all-cause hospitalization and allcause mortality. There was no difference in 
FVC change, six minute walk distance (6MWD), or diffusing capacity of the lung for carbon monoxide $\left(\mathrm{DL}_{\mathrm{CO}}\right)$ for the survivors ${ }^{27}$.

\section{Phosphodiesterase-5 inhibitor}

The Sildenafil Trial of Exercise Performance in Idiopathic Pulmonary Fibrosis (STEP-IPF) in patients with advanced disease $\left(\mathrm{DL}_{\mathrm{CO}}<35 \%\right.$ predicted) demonstrated no difference in the proportion of patients with at least a $20 \%$ improvement in 6MWD after taking sildenafil $20 \mathrm{mg}$ three times a day for 12 weeks compared to placebo ${ }^{28}$. However, there were numerous secondary endpoints with a positive treatment effect relating to gas exchange and quality of life. In a post hoc subgroup analysis of 119 participants with echocardiogram results, those with right ventricular systolic dysfunction (RVSD) who were treated with sildenafil experienced significantly less decline in 6MWD as well as small benefits in quality of life questionnaire scores ${ }^{29}$. Two studies are under way to assess the impact of sildenafil in addition to anti-fibrotic therapy in IPF patients with advanced disease (ClinicalTrials.gov Identifier: NCT02802345 for nintedanib and NCT02951429 for pirfenidone).

\section{SUMMARY OF THE PREVIOUS ERA}

Based on the evidence summarized thus far, the most recent ATS/ERS/Japanese Respiratory Society (JRS)/Latin American Thoracic Association (ALAT) clinical practice guideline on IPF treatment strongly recommends against the use of anticoagulation, combination
Table 1. Summary of American Thoracic Society 2015 Clinical Practice Guideline Update ${ }^{3}$

\begin{tabular}{|l|l|}
\hline Therapy & Recommendation \\
\hline Anticoagulation (warfarin) & $\begin{array}{c}\text { Strong recommendation } \\
\text { against use }\end{array}$ \\
\hline $\begin{array}{l}\text { Prednisone/azathioprine/ } \\
\text { N-acetylcysteine }\end{array}$ & $\begin{array}{c}\text { Strong recommendation } \\
\text { against use }\end{array}$ \\
\hline Ambrisentan & $\begin{array}{c}\text { Strong recommendation } \\
\text { against use }\end{array}$ \\
\hline Imatinib & $\begin{array}{c}\text { Strong recommendation } \\
\text { against use }\end{array}$ \\
\hline Macitentan/bosentan & $\begin{array}{c}\text { Conditional recommendation } \\
\text { against use }\end{array}$ \\
\hline Sildenafil & $\begin{array}{c}\text { Conditional recommendation } \\
\text { against use }\end{array}$ \\
\hline N-acetylcysteine & $\begin{array}{c}\text { Conditional recommendation } \\
\text { against use }\end{array}$ \\
\hline
\end{tabular}

This guideline update also included conditional recommendations for use of pirfenidone and nintedanib.

prednisone/azathioprine/NAC, ambrisentan, and imatinib. Conditional recommendations against the use of dual endothelin receptor antagonists (macitentan and bosentan), sildenafil, and NAC were also issued (Table 1) ${ }^{3}$.

\section{CURRENT ERA: CLINICAL PRACTICE GUIDELINES}

A welcome breakthrough after the long succession of failures in therapeutic drug trials in IPF came with more recent clinical trials of pirfenidone and nintedanib.

\section{Pirfenidone}

Pirfenidone has pleiotropic anti-inflammatory and anti-fibrotic effects, but its exact mechanism is unknown. In animal models, it reduced pro-inflammatory cytokines such as 
TNF- $\alpha$, TGF- $\beta$, fibroblast proliferation and myofibroblast differentiation ${ }^{30,31}$. In a phase 2 , open label study for terminally ill patients with advanced IPF, pirfenidone appeared to stabilize lung function and reduce conventional therapy (steroids and immunosuppressive medication) usage $^{32}$. The first randomized controlled trial of pirfenidone suggested a reduction in acute exacerbations of IPF as a secondary endpoint ${ }^{33}$. A subsequent study demonstrated that high dose $(1,800 \mathrm{mg} /$ day $)$ pirfenidone decreased the rate of decline in vital capacity (primary endpoint) and increased progression-free survival over 52 weeks $^{34}$. This study was criticized for a highly selective enrolment and a mid-study change in the primary endpoint after blinded interim analysis. Nevertheless, these studies sparked renewed interest in pirfenidone.

\section{The CAPACITY (Clinical Studies Assessing} Pirfenidone in Idiopathic Pulmonary Fibrosis: Research of Efficacy and Safety Outcomes) program included two concurrent large, randomized, placebo-controlled multinational trials of pirfenidone for IPF. There was a significant benefit in the change in percentage of the predicted FVC in patients with mild to moderate IPF receiving high dose $(2,403 \mathrm{mg} /$ day) pirfenidone compared to placebo in one study $(-8.0$ versus $-12.4 \%$; $p=0.001)$ but not the second ( -9.0 versus $-9.6 \%$; $=0.501)$. The pre-specified pooled analysis suggested attenuation in physiological progression with pirfenidone therapy. Patients in the pirfenidone group reported a higher incidence of nausea, dyspepsia, photosensitivity and dizziness ${ }^{35}$. Based on the results of these trials, multiple countries approved pirfenidone for the treatment of mild to moderate IPF. After reviewing cost-effectiveness comparisons, the National
Institute for Health and Care Excellence (NICE) supported pirfenidone as a therapeutic option in IPF patients with an FVC between $50 \%$ and $80 \%$ predicted $^{36}$.

Because of the inconsistency between the two studies in the CAPACITY program, United States regulatory authorities requested an additional trial to support the approval of pirfenidone. Assessment of pirfenidone to Confirm Efficacy and Safety in Idiopathic Pulmonary Fibrosis (ASCEND) modified the design of CAPACITY to include strict centralized procedures for diagnosis, spirometry, and adjudication of deaths. The primary endpoint of change in percentage predicted FVC was met at 52 weeks with a $45.1 \%$ relative reduction in FVC decline in the pirfenidone group compared to placebo ( $p<0.001)$. Secondary endpoints showed a relative risk reduction in the composite endpoint of death or disease progression by $43 \%$ in the pirfenidone group ( $p<0.001)$, but no significant difference in all-cause mortality or dyspnoea score ${ }^{37}$. The totality of the data and internal consistency between physiological surrogates and other markers of disease progression compelled the Food and Drug Administration to approve pirfenidone for use in IPF patients ${ }^{38}$.

A pre-specified pooled analysis of the CAPACITY and ASCEND trials with high dose pirfenidone confirmed a treatment benefit with pirfenidone in the composite endpoints of FVC decline or death, progression free survival, and 6MWD decrement or death. There was a statistically significant reduction in all-cause mortality (hazard ratio [HR]: 0.52, 95\%; confidence interval [CI]: 0.31-0.87) ${ }^{39}$. Additional analyses of these data plus prior Japanese randomized controlled trials further support 
a possible benefit on the risk of mortality over 120 weeks of therapy ${ }^{40}$.

Given the duration of availability of pirfenidone and extensive patient exposure, additional relevant data have become available. Extension studies of the original pivotal trials and prospective registry data have suggested tolerability similar to that of the randomized trials with predominantly gastrointestinal and skin adverse events. Dose adjustment has been associated with better tolerance ${ }^{41,42}$. Long term follow-up data have confirmed these findings ${ }^{43}$.

Analyses from the CAPACITY and ASCEND trials suggest substantial intra-subject heterogeneity in longitudinal FVC data ${ }^{44}$. A retrospective analysis of patients from Giessen and Turin provides additional insights, albeit limited by lack of randomization. In a broader population than included in traditional clinical trials, heterogeneity in treatment response was evident with the largest group demonstrating stability after a previous period of instability (Fig. 1) ${ }^{45}$.

\section{Nintedanib}

An initial phase 2 clinical trial (TOMORROW) demonstrated that a tyrosine kinase inhibitor, nintedanib, reduced FVC decline and acute exacerbations in patients with mild to moderate IPF ${ }^{46}$. This was confirmed in two phase 3 randomized placebo-controlled trials INPULSIS-1 and INPULSIS-2. Inclusion criteria for the diagnosis of IPF were broader than in the pirfenidone trials; patients with high resolution computed tomography (HRCT) scans showing possible usual interstitial pneumonia (UIP) were included without the requirement of surgical lung biopsy to confirm diagnosis. The primary endpoint of annual rate of change in FVC favoured nintedanib over placebo in both studies $(-114.7 \mathrm{ml}$ with nintedanib versus $-239.9 \mathrm{ml}$ with placebo in INPULSIS-1, p < 0.001; -113.6 ml with nintedanib versus $-207.3 \mathrm{ml}$ with placebo in INPULSIS-2, $\mathrm{p}<0.001$ ). There was an inconsistent effect on acute exacerbations and Saint Georges Respiratory Questionnaire (SGRQ) score observed in the two trials and no difference in mortality (respiratory or all-cause) ${ }^{47}$. Importantly, the beneficial effects of nintedanib were seen across a broad range of patient subgroups ${ }^{48}$, including subgroups of adjudicated IPF defined by honeycombing on HRCT and/or UIP on lung biopsy versus no honeycombing and no lung biopsy ${ }^{49}$ (Fig. 2). This finding implies that the natural history of disease progression and response to therapy may be similar in patients with or without definite UIP pattern on HRCT or lung biopsy.

Results from the individual nintedanib studies and pooled analyses confirm that gastrointestinal side effects are the most frequent adverse events noted ${ }^{50}$. Diarrhoea was the most frequently noted event although only $4.4 \%$ of nintedanib-treated patients discontinued the trial medication as a result of this event. The gastrointestinal adverse events can generally be managed with anti-motility agents and sometimes require dose adjustments. Like with pirfenidone, monitoring of liver enzymes before and periodically during treatment is recommended. Arterial thromboembolic events occurred in $2.5 \%$ of nintedanib and $0.8 \%$ of placebo patients. Myocardial infarction was the most common of these events, reported in 


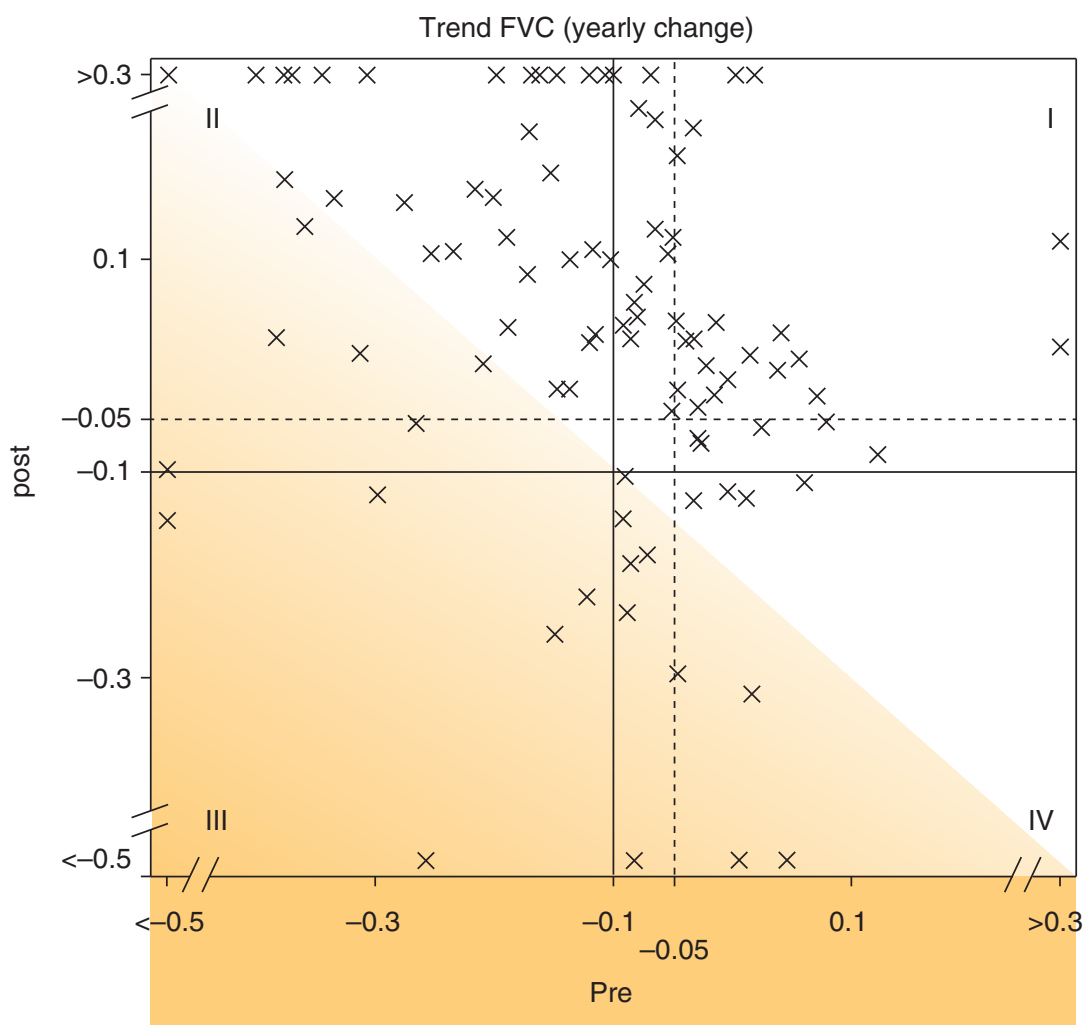

\begin{tabular}{|l|c|c|c|}
\hline & $\begin{array}{c}\text { Annual decline } \\
\text { of FCV with a cut-off } \\
\text { value of 10\% }\end{array}$ & $\begin{array}{c}\text { Annual decline } \\
\text { of FVC with a cut-off } \\
\text { value of 5\% }\end{array}$ \\
\hline Stable-stable & I & $37(38.1 \%)$ & $20(20.6 \%)$ \\
\hline Unstable-stable & II & $40(41.2 \%)$ & $51(52.6 \%)$ \\
\hline Unstable-unstable & III & $6(6.2 \%)$ & $13(13.4 \%)$ \\
\hline Stable-unstable & IV & $14(14.4 \%)$ & $13(13.4 \%)$ \\
\hline
\end{tabular}

FIGURE 1. Stability of yearly FVC change before and after pirfenidone treatment. Each $\mathrm{X}$ represents an individual data pair for the FVC change with resulting subgroups of I (stable before-stable after); II (unstable before-stable after); III (unstable before-unstable after); IV (stable before-unstable after). The majority of patients achieved disease stability after treatment (79.3\%) but a significant minority (20.6\%) experienced disease progression (reproduced with permission from Loeh B et al. ${ }^{45}$ ).

FVC: forced vital capacity.

$1.6 \%$ of nintedanib and $0.5 \%$ of placebo patients ${ }^{50}$. Caution is advised in patients with known coronary artery disease and treatment interruption is recommended for myocardial ischemia.

\section{SUMMARY OF THE CURRENT ERA}

Based on the evidence presented above, the most recent ATS/ERS/JRS/ALAT clinical practice 


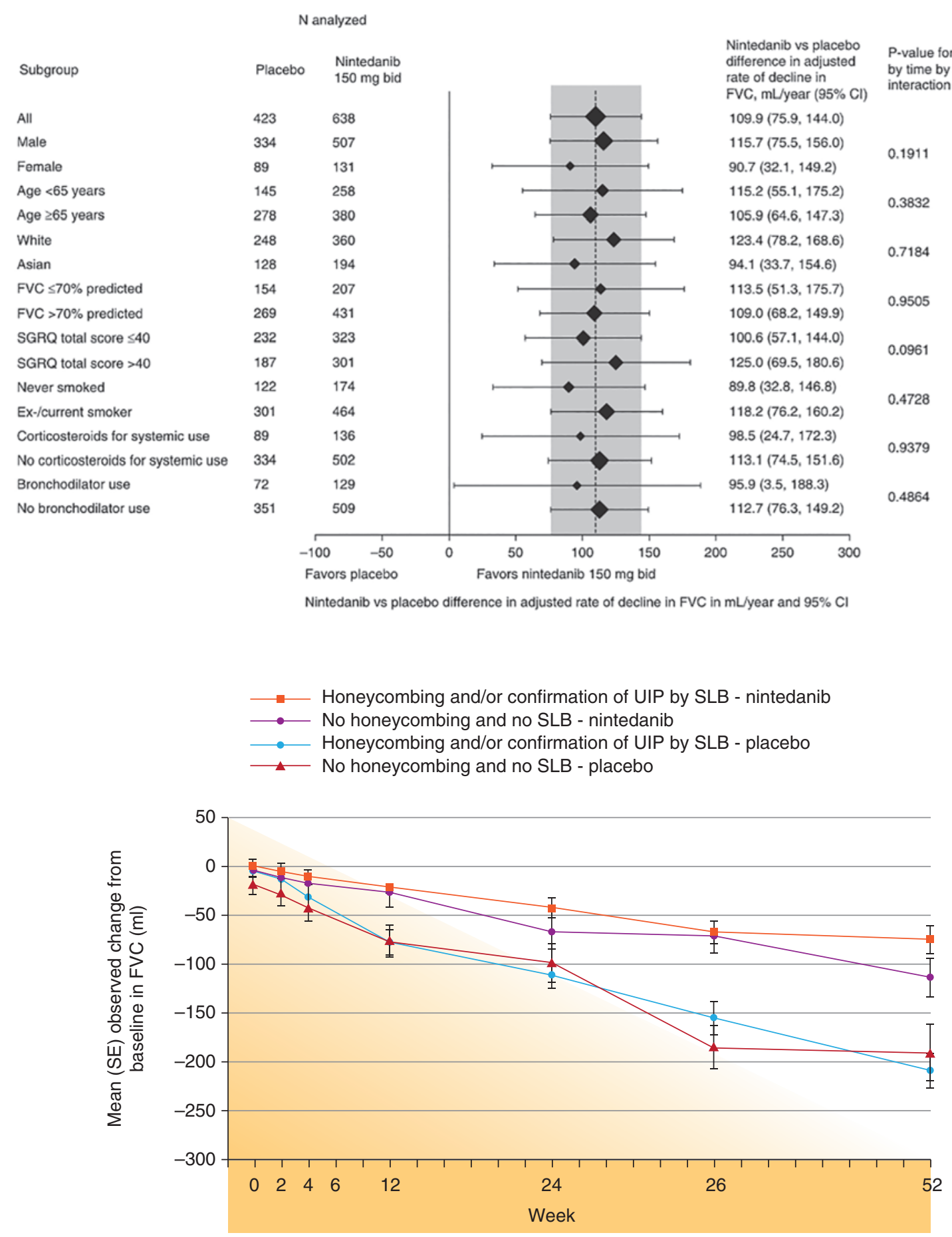

FIGURE 2. Effect of nintedanib on change from baseline in FVC not affected by subgroup when analyzed by presence of honeycombing, demographics, or pre-treatment percentage predicted FVC (reproduced with permission from Costabel $U$ et al. ${ }^{48}$ and Raghu $G$ et al. ${ }^{49}$.

FVC: forced vital capacity; SGRQ: Saint Georges Respiratory Questionnaire; SLB: surgical lung biopsy. 
Treatment 1 vs treatment 2

Pirfenidone vs imatinib

Pirfenidone vs placebo

Nintedanib vs imatinib

Nintedanib vs placebo

Pirfenidone vs nintedanib

Pirfenidone vs imatinib $95 \% \mathrm{Cl}(0.05564-0.5667)$

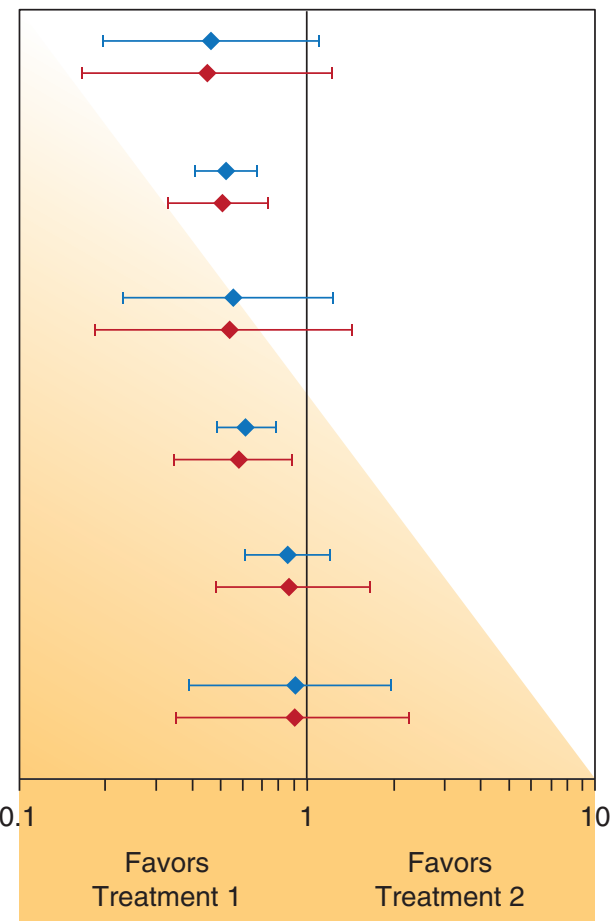

$0.52(0.41-0.67)$

$0.51(0.33-0.74)$

$0.55(0.23-1.24)$

$0.53(0.18-1.43)$

$0.61(0.48-0.78)$

$0.58(0.34-0.89)$

$0.86(0.60-1.20)$

$0.87(0.48-1.66)$

$0.91(0.39-1.97)$

$0.90(0.35-2.26)$

$\longmapsto$ Fixed effects

$\longmapsto$ Random effects (Informative Prior)

Figure 3. Forest plot of results of network meta-analysis for pairwise comparisons of $\% F V C$ decline by $\geq 10 \%$. Pirfenidone and nintedanib have similar effects on lung function (reproduced with permission from Canestaro WJ et al. ${ }^{52}$ ).

Cl: confidence interval; FVC: forced vital capacity; OR: odds ratio.

guideline on IPF treatment conditionally recommends for the use of pirfenidone and nintedanib $^{3}$. As there are no head to head comparisons between the two agents it is not possible to recommend one over the other. Interestingly, two complex statistical network analyses have recently been published contrasting results from clinical trials of multiple agents $^{51,52}$. These analyses have reached similar conclusions that both agents likely result in similar clinical and physiological results (Fig. 3). The availability of two effective agents with differing dosing regimens and tolerability profiles provides the patient and clinician with valuable therapeutic options that must be personalized ${ }^{53}$. The timing of initiation of therapy remains controversial ${ }^{54}$. It appears that their benefit is seen across a broad spectrum of patients with mild to moderate spirometric severity and, in the case of nintedanib, across various HRCT patterns ${ }^{55-57}$. As such, discussion with the patient regarding the potential benefit of early intervention seems appropriate. 


\section{FUTURE ERA}

Though the available agents do not provide a "cure", future trials must now be designed with this new standard of care in mind, calling into question the ethics of placebo-controlled studies ${ }^{58}$. This natural progression lends itself to combination therapy studies (the possible combination of pirfenidone and nintedanib or an investigational drug added to either pirfenidone or nintedanib) as well as non-inferiority and superiority studies in late phase clinical trials ${ }^{59}$. To add to this challenge, no well-designed studies have been able to consistently show that any drug significantly improves mortality or quality of life in IPF. Unfortunately, the low event rate of meaningful clinical endpoints like mortality would require trials to enroll 2,582 IPF patients and follow them for up to 5 years to detect a $25 \%$ reduction in mortality with $90 \%$ power. This would cost approximately $\$ 250$ million, making the adoption of allcause mortality as a primary endpoint for IPF clinical trials impractical and cost-prohibitive ${ }^{60}$.

\section{Combination therapy}

Given the pleiotropic and complex nature of disease pathogenesis, it seems logical that combination therapy will become a clinical norm ${ }^{59}$. Perhaps, the most intuitive place to begin studying combination therapy is with the two drugs already shown to slow the progression of disease in IPF. Several ongoing studies are evaluating the safety and tolerability of pirfenidone in combination with nintedanib and plasma drug concentrations when the two are administered together
(NCT02598193, NCT02579603, NCT02606877). So far, a small phase 2 clinical trial has shown no serious adverse events with the combination, but a decrease in the bioavailability of nintedanib when taken with pirfenidone may be a limiting factor ${ }^{61}$. As noted earlier, the randomised, double-blind, placebo-controlled, Phase 2 study that investigated the use of NAC in combination with pirfenidone in IPF (PANORAMA) trial examined the safety and tolerability of NAC or placebo in addition to pirfenidone for IPF treatment ${ }^{10}$.

\section{Monoclonal antibodies}

Several cell signalling pathways have been implicated in the pathogenesis of IPF and provide targets for new drug development. Connective tissue growth factor (CTGF), a protein that participates in cell adhesion and migration, angiogenesis and extracellular matrix deposition, has increased expression in the lung fibroblasts of IPF patients ${ }^{62}$ and is required for TGF- $\beta$-mediated lung fibrosis. CTGF antibody FG-3019 has been found to be effective at attenuating lung collagen deposition in a murine bleomycin model of pulmonary fibrosis (NCT01890265) ${ }^{63}$ and is now being tested in a phase 2 randomized double-blind placebo controlled trial to assess its safety and efficacy in treating IPF after an open label study demonstrated no concerns with safety or tolerability (NCT01262001) ${ }^{64}$.

Interleukin-13 (IL-13), a Th2 cytokine, plays a role in promoting fibroblast collagen production and myofibroblast differentiation ${ }^{65}$. Its concentration is also increased in IPF bronchoalveolar lavage fluid (BALF) ${ }^{66}$. Two IL-13 antibodies are currently undergoing phase 2 
trials to investigate safety and tolerability in IPF treatment: lebrikizumab and SAR156597 with a third (tralokinumab) study recently terminated after interim analysis demonstrated lack of efficacy (NCT01872689, NCT01529853, and NCT01629667 respectively).

TGF- $\beta$ has long been recognized as a key pro-fibrotic mediator in lung fibrosis along with its many other functions in cell proliferation, apoptosis and immune regulation ${ }^{67}$. The discovery of $\alpha \mathrm{V} \beta 6$ integrin and its activation of latent TGF- $\beta$ after epithelial injury and inflammation $^{68}$ led to the development of a humanized monoclonal antibody BG00011 (formerly known as STX-100). A phase 2 trial is now recruiting IPF patients to study safety/ tolerability, pharmacokinetic parameters, and change in peripheral blood and BALF biomarkers (NCT01371305).

\section{Antimicrobial therapy}

Growing evidence points to the role of lung microbial dysbiosis as a contributor to chronic lung disease and exacerbations ${ }^{69}$. Correlating Outcomes with biochemical Markers to Estimate Time-progression in Idiopathic Pulmonary Fibrosis (COMET-IPF) investigators documented an association between IPF disease progression and relative abundance of Streptococcus and Staphylococcus in the BALF of IPF patients ${ }^{70}$. Subsequent work from this group linked this alteration in the lung microbial community to many biological pathways associated with disease progression in IPF using comprehensive association pathway analyses ${ }^{71}$. Peripheral blood mononuclear cell gene expression, lung fibroblast tolllike receptor 9 expression, and circulating leukocyte phenotypes reflected down regulation of immune response pathways that were associated with IPF progression-free survival and alteration in the lung microbial community (Fig. 4). Another group confirmed these findings reporting that IPF patients had double the burden of bacteria (specifically Haemophilus, Streptococcus, Neisseria, and Veillonella) in their BALF compared to healthy control subjects $^{72}$. In an integrated analysis they have also linked the lung microbial alteration to host defense response ${ }^{73}$. Importantly, a small study compared co-trimoxazole with placebo in addition to usual care demonstrating a significant reduction in all-cause mortality (HR: 0.21; 95\% CI: 0.06-0.78; $\mathrm{p}=0.02$ ) in a per-protocol analysis ${ }^{74}$. Based in part on these findings, several studies of antimicrobial therapy are ongoing including the Clinical Efficacy of Antimicrobial Therapy Strategy Using Pragmatic Design in IPF (CleanUP IPF) (NCT02759120), the Efficacy and Mechanism Evaluation of Treating Idiopathic Pulmonary Fibrosis with the Addition of Co-trimoxazloe (EME-TIPAC) (EudraCT 2014-004058-32) and a pilot study of trimethoprim-sulfamethoxazole in IPF (EudraCT 2012-005409-38).

\section{Stem cell therapy}

Several pre-clinical studies using the murine bleomycin model of IPF have suggested that mesenchymal stem cell therapy may be useful in reducing pro-inflammatory cytokines, neutrophilic infiltration, and collagen deposition ${ }^{75}$. A small phase I study of bone-marrow derived mesenchymal stem cell infusion in mild to moderate IPF patients showed no treatment related serious adverse events ${ }^{76}$, but there remain some concerns that stem cells 
A

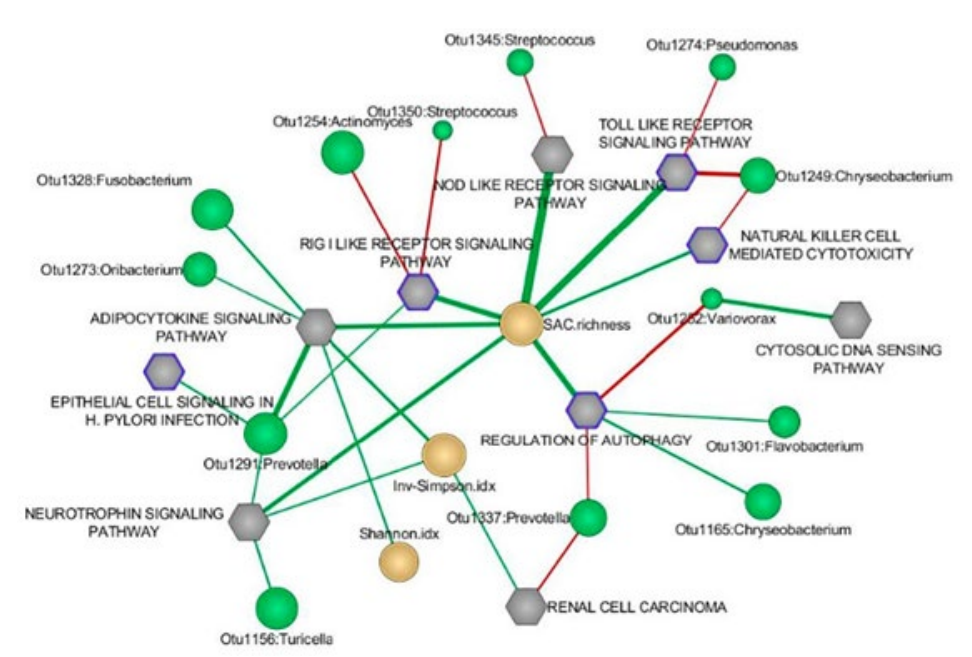

B

Correlation of host gene modules with clinical traits and microbial community

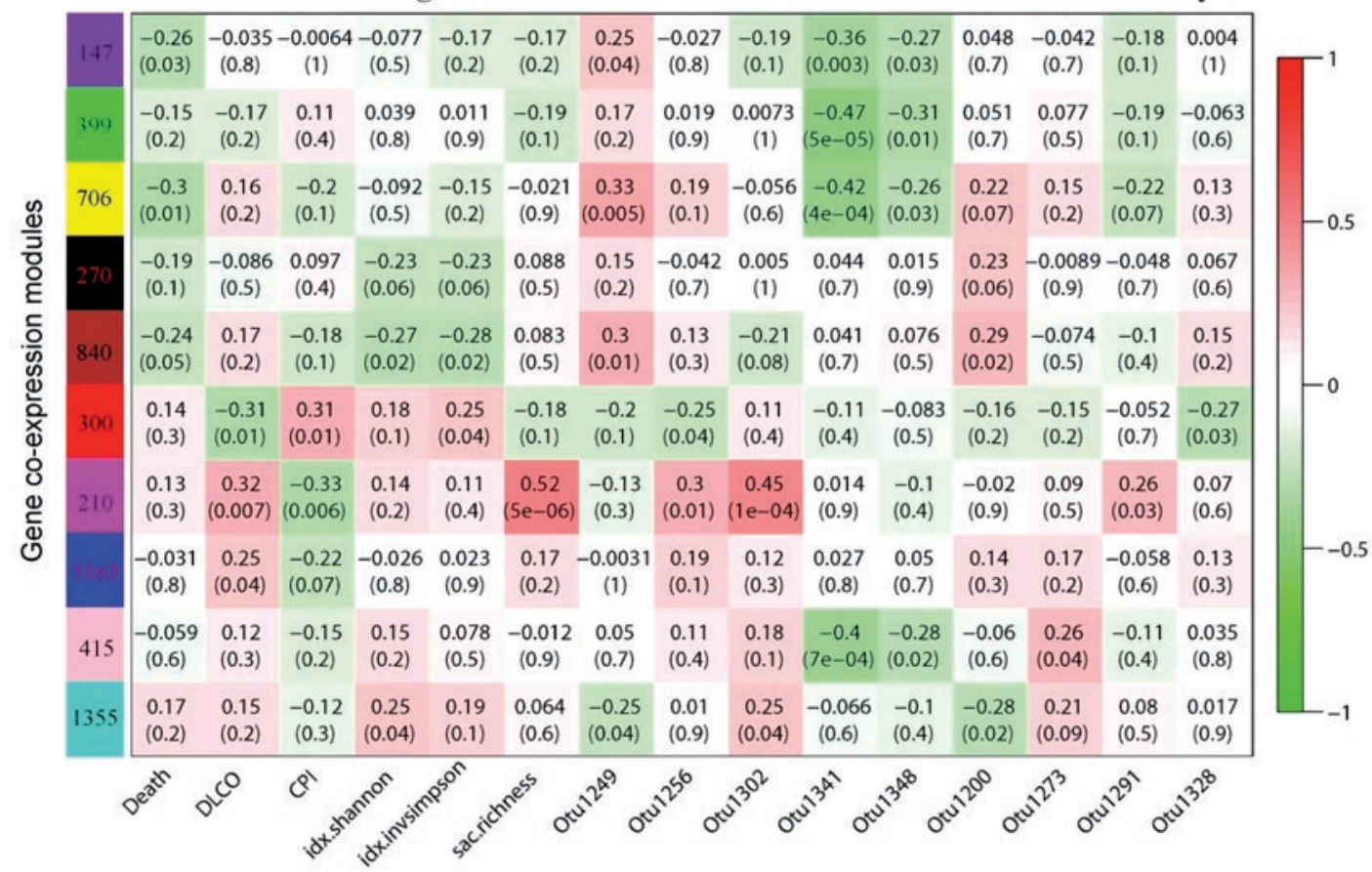

Figure 4.

A. Correlation interaction of PFS-associated host canonical pathways (top). Ten canonical pathways involving the immune inflammatory response and pathogen infection or pattern recognition receptors (grey hexagons) correlated with microbial community features (green circles). Golden circles represent microbial diversity indices and green circles demonstrate 0TU abundance with the diameter of the circle proportional to the correlation coefficient. Red lines represent positive correlation and green lines represent negative correlation, with the thickness of the lines determined by 1-(p-value).

B. Correlation of host gene modules with clinical traits and microbial community (bottom). A red box represents positive correlation while a green box represents negative correlation. For example, the magenta module 210 is positively correlated with $\mathrm{DL}_{\mathrm{co}}, 0 \mathrm{TU} 1302$ (Pseudomonadaceae), 0TU1256 (Prevotella), and 0TU1291 (Prevotella) (reproduced with permission from Huang $S$ et al. ${ }^{71}$ ).

PFS: progression free survival; OTU: operational taxonomic unit; $\mathrm{CPI}$ : composite physiologic index; $\mathrm{DL}_{\mathrm{CO}}$ : diffusing capacity of the lung for carbon monoxide. 
may contribute to the population of abnormal fibroblasts or even encourage malignant transformation and larger long-term follow up studies are needed ${ }^{75}$.

\section{Precision medicine}

IPF is a highly heterogeneous disease with variable clinical course, potential risk factors, and associated co-morbidities. It is likely that this reflects multiple biological processes driven by genetic/molecular, environmental and behavioural influences ${ }^{77,78}$. NIH's Precision Medicine Initiative Working Group defines precision medicine as an approach to disease treatment and prevention that seeks to maximize effectiveness by incorporating individual variability in genes, environment, and lifestyle $\mathrm{e}^{79}$. Most precision medicine discussion to date has focused on molecular and genetic markers in identifying distinct endotypes ${ }^{77}$. There are numerous groups developing molecular markers that are linked to clinically relevant features and potential therapeutic $\operatorname{targets}^{78}$. A recent, albeit disappointing, approach was published in the targeting of lysyl oxidase-like 2 (LOXL2) with a monoclonal antibody, simtuzumab ${ }^{80}$. The overall results were negative as were those predefined by circulating LOXL2 concentrations (Fig. 5). These results may reflect challenges with the underlying antibody, redundancy in pro-fibrotic pathways, or the difficulties in IPF clinical studies. Nevertheless, this sentinel study provides a roadmap for the design and conduct of clinical studies targeting promising biological pathways and including relevant, companion diagnostics. In fact, the ongoing antimicrobial therapy studies described earlier have included similar, robust biomarker approaches to advance the potential of personalized therapy to IPF patients.

\section{Conclusion}

Idiopathic pulmonary fibrosis is a complex and heterogeneous disease, creating formidable challenges for effective treatment. Over the last three decades, there has been a paradigm shift from immunosuppressive strategies to pleiotropic anti-fibrotic treatments with resulting efficacious therapies, a first in this challenging field. The future will likely move toward targeted therapy, combinatorial approaches and precision medicine based on biomarker and gene expression profiling (Fig. 6). Despite earlier disappointing results and trial design difficulties, recent successes have renewed excitement and optimism for the future of IPF therapy.

\section{CONFLICT OF INTEREST}

Dr. Fernando J. Martínez reports non-financial support from Bayer, Gilead, Nitto, Patara, and Biogen/Stromedix; personal fees, non-financial support and other from Boehringer Ingelheim, Centocor, personal fees from Genentech, Bellerophon (formerly Ikaria), Kadmon, Nycomed/Takeda, Pfizer, Veracyte, the American Thoracic Society, Academic CME, MedEd Consulting, National Association for Continuing Education, Axon Communication, Johnson \& Johnson, Clarion Communications, Potomac Center for Medical Education (PCME), Merck (formerly Afferent), Adept Field Solutions (London, UK); and grants from National Institutes of Health. Dr. Xiaoping Wu and Dr. Robert J. Kaner have nothing to disclose. 


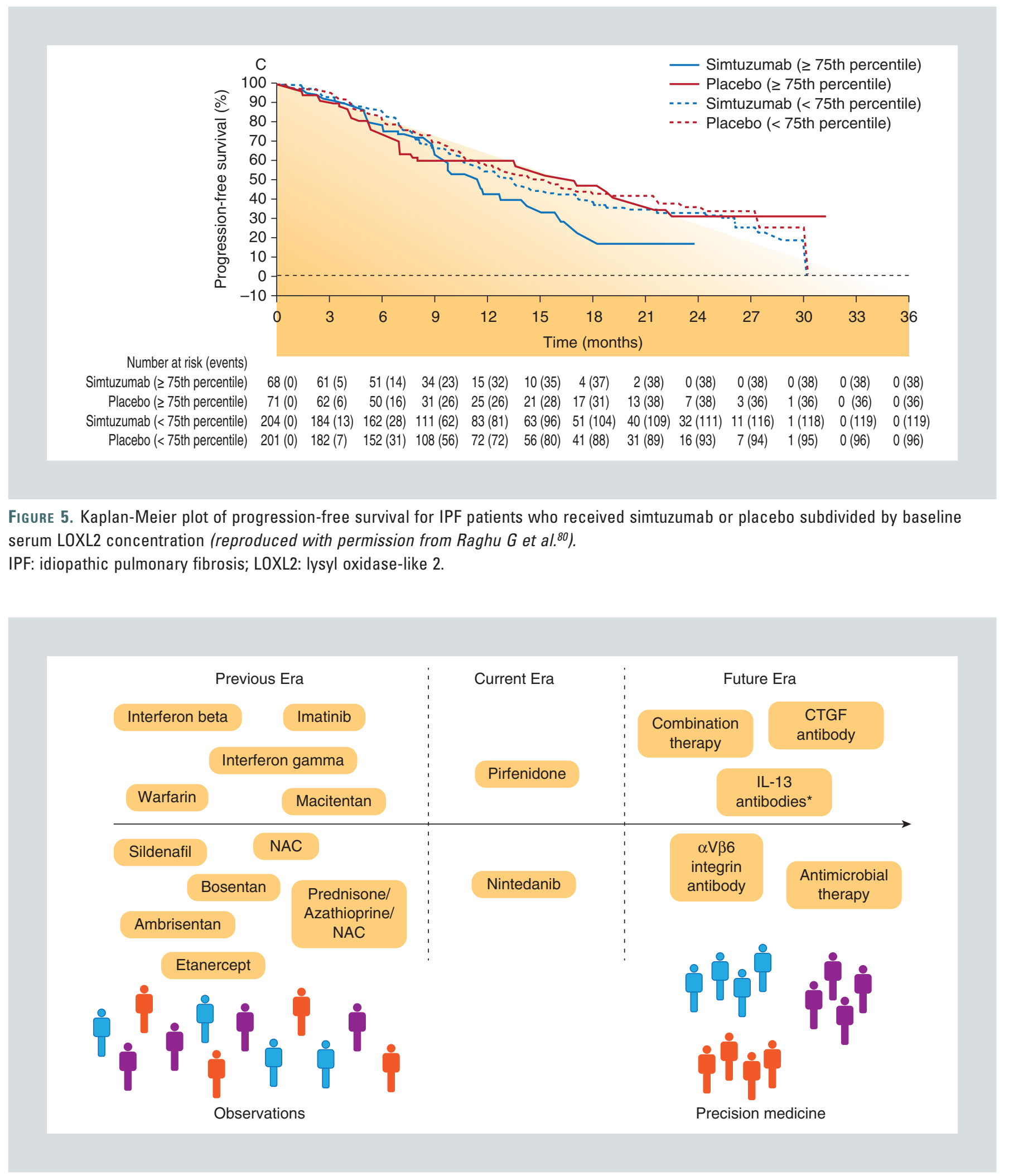

FIgURE 6. Summary of IPF therapy studies — past, present, and future.

*Tralokinumab trial recently terminated and 2 other trials ongoing.

CTGF: connective tissue growth factor; IL-13: interleukin 13; IPF: idiopathic pulmonary fibrosis; LOXL2: Iysyl oxidase-like 2;

NAC: N-acetylcysteine. 


\section{REFERENCES}

1. Raghu G, Collard HR, Egan JJ et al. An official ATS/ERS/JRS/ALAT statement: idiopathic pulmonary fibrosis: evidence-based guidelines for diagnosis and management. Am J Respir Crit Care Med. 2011;183:788-824.

2. Hutchinson J, Fogarty A, Hubbard R, McKeever T. Global incidence and mortality of idiopathic pulmonary fibrosis: a systematic review. Eur Respir J. 2015;46:795-806.

3. Raghu G, Rochwerg B, Zhang Y et al. An Official ATS/ERS/JRS/ALAT Clinical Practice Guideline: Treatment of Idiopathic Pulmonary Fibrosis. An Update of the 2011 Clinical Practice Guideline. Am J Respir Crit Care Med. 2015;192:e3-e19.

4. American Thoracic Society. Idiopathic pulmonary fibrosis: diagnosis and treatment: international consensus statement. Am J Respir Crit Care Med. 2000; $161: 646-64$.

5. Winterbauer RH, Hammar SP, Hallman KO et al. Diffuse interstitial pneumonitis. Clinicopathologic correlations in 20 patients treated with prednisone/azathioprine. Am J Med. 1978;65:661-72.

6. Raghu G, Depaso WJ, Cain K et al. Azathioprine Combined with Prednisone in the Treatment of Idiopathic Pulmonary Fibrosis: A Prospective Double-blind Randomized, Placebo-controlled Clinical Trial. Am Rev Respir Dis. 1991;144:291-6.

7. Johnson MA, Kwan S, Snell NJ, Nunn AJ, Darbyshire JH, Turner-Warwick M. Randomised controlled trial comparing prednisolone alone with cyclophosphamide and low dose prednisolone in combination in cryptogenic fibrosing alveolitis. Thorax. 1989; 44:280-8.

8. Raghu G, Anstrom KJ, King TE Jr et al. Idiopathic Pulmonary Fibrosis Clinical Research Group. Prednisone, Azathioprine, and N-Acetylcysteine for Pulmonary Fibrosis. N Engl J Med. 2012;366:1968-77.

9. Martinez FJ, de Andrade JA, Anstrom KJ et al. Idiopathic Pulmonary Fibrosis Research Group. "Randomized trial of acetylcysteine in idiopathic pulmonary fibrosis." N Engl J Med. 2014;370:2093-2101.

10. Behr J, Bendstrup E, Crestani et al. Safety and tolerability of acetylcysteine and pirfenidone combination therapy in idiopathic pulmonary fibrosis: a randomized, double-blind, placebo-controlled, phase 2 trial. Lancet Respir Med. 2016;4:445-53.

11. Oldham JM, Ma SF, Martinez FJ et al. TOLLIP, MUC5B, and the Response to NAcetylcysteine among Individuals with Idiopathic Pulmonary Fibrosis. Am J Respir Crit Care Med. 2015;192:1475-82.

12. Raghu G, Bozic C, Brown $K$ et al. Feasibility of a trial of interferon beta-1A (IFN- Beta-1A) in the treatment of Idiopathic Pulmonary Fibrosis. Am J Respir Crit Care Med. 2001;163:A707.

13. Ziesche R, Hofbauer E, Willmann K, Petkov V, Block LH. A preliminary study of longterm treatment with interferon gamma- $1 \mathrm{~b}$ and low-dose prednisolone in patients with idiopathic pulmonary fibrosis. N Engl J Med. 1999;341:1264-9.

14. Raghu G, Brown KK, Bradford WZ et al. Idiopathic Pulmonary Fibrosis Study Group. A Placebo-Controlled Trial of Interferon Gamma- $1 \mathrm{~b}$ in Patients with Idiopathic Pulmonary Fibrosis. N Engl J Med. 2004;350:125-33.

15. King TE Jr, Albera C, Bradford WZ et al. Effect of interferon gamma-1b on survival in patients with idiopathic pulmonary fibrosis (INSPIRE): a multicenter, randomized, placebo-controlled trial. The Lance.t 2009;374:222-8.

16. Piguet PF, Ribaux C, Karpuz V, Grau GE, Kapanci Y. Expression and localization of tumour necrosis factor-alpha and its mRNA in idiopathic pulmonary fibrosis. Am J Pathol. 1993;143:651-5.

17. Piguet PF, Vesin C. Treatment by human recombinant soluble TNF receptor of pulmonary fibrosis induced by bleomycin or silica in mice. Eur Respir J. 1994;7:515- 8 .

18. Raghu G, Brown KK, Costabel U et al. Treatment of Idiopathic Pulmonary Fibrosis with Etanercept. An Exploratory, Placebo-controlled Trial. Am J Respir Crit Care Med. 2008;178:948-55.

19. Daniels CE, Lasky JA, Limper AH, Mieras K, Gabor E, Schroeder DR. Imatinib-IPF Study Investigators. Imatinib treatment for idiopathic pulmonary fibrosis: Randomized placebo-controlled trial results. Am J Respir Crit Care Med. 2010;181:604-10.

20. Giaid A, Michel RP, Stewart DJ, Sheppard M, Corrin B, Hamid Q. Expression of endothelin- 1 in lungs of patients with cryptogenic fibrosing alveolitis. Lancet. 1993;341:1550-4.

21. King TE Jr, Behr J, Brown KK et al. BUILD-1: a randomized placebo-controlled trial of bosentan in idiopathic pulmonary fibrosis. Am J Respir Crit Care Med. 2008;177:75-81.

22. King TE Jr, Brown KK, Raghu G et al. BUILD-3: a randomized, controlled trial of bosentan in idiopathic pulmonary fibrosis. Am J Respir Crit Care Med. 2011;184:92-9.

23. Raghu G, King TE Jr, Behr J et al. Impact of bosentan on health-related quality of life and dyspnoea in idiopathic pulmonary fibrosis: the BUILD-1 trial. Eur Respir J. 2010;35:118-23.

24. Raghu G, Million-Rousseau R, Morganti A, Perchenet L, Behr J. MUSIC Study Group. Macitentan for the treatment of idiopathic pulmonary fibrosis: the randomised controlled MUSIC trial. Eur Respir J. 2013;42: 1622-32.

25. Raghu G, Behr J, Brown KK et al. ARTEMIS-IPF Investigators. Treatment of idiopathic pulmonary fibrosis with ambrisentan: a parallel, randomized trial. Ann Intern Med. 2013;158:641-9.

26. Kubo H, Nakayama K, Yanai M et al. Anticoagulant therapy for idiopathic pulmonary fibrosis. Chest. 2005;128:1475-82.

27. Noth I, Anstrom KJ, Calvert SB et al. Idiopathic Pulmonary Fibrosis Clinical Research Network. A placebo-controlled randomized trial of warfarin in idiopathic pulmonary fibrosis. Am J Respir Crit Care Med. 2012;186: 88-95.

28. Zisman DA, Schwarz M, Anstrom KJ, Collard HR, Flaherty KR, Hunninghake GW. Idiopathic Pulmonary Fibrosis Clinical Research Network. A Controlled Trial of Sildenafil in Advanced Idiopathic Pulmonary Fibrosis. N Engl J Med. 2010;363:620-8.

29. Han MK, Bach DS, Hagan PG et al. IPFnet Investigators. Sildenafil Preserves Exercise Capacity in Patients with Idiopathic Pulmonary Fibrosis and Right-sided Ventricular Dysfunction. Chest. 2013;143:1699-1708.

30. Oku H, Shimizu T, Kawabata T et al. Antifibrotic action of pirfenidone and prednisolone: different effects on pulmonary cytokines and growth factors in bleomycin induced murine pulmonary fibrosis. Eur J Pharmacol. 2008 590:400-8.

31. Ahluwalia N, Shea BS, and Tager AM. New Therapeutic Targets in Idiopathic Pulmonary Fibrosis. Aiming to Rein in Runaway Would-Healing Responses. Am J Respir Crit Care Med. 2014;190:867-78.

32. Raghu G, Johnson WC, Lockhart D, Mageto Y. Treatment of Idiopathic Pulmonary Fibrosis with a New Antifibrotic Agent, Pirfenidone. Results of a Prospective, Open-label Phase II Study. Am J Respir Crit Care Med. 1999;159:1061-9.

33. Azuma A, Nukiwa T, Tsuboi E et al. Double-blind, placebo-controlled trial of pirfenidone in patients with idiopathic pulmonary fibrosis. Am J Respir Crit Care Med. 2005;171:1040-7.

34. Taniguchi H, Ebina M, Kondoh Y, et al. Pirfenidone in idiopathic pulmonary fibrosis. Eur Respir J. 2010;35:821-9.

35. Noble PW, Albera C, Bradford WZ et al; CAPACITY Study Group. Pirfenidone inpatients with idiopathic pulmonary fibrosis (CAPACITY): two randomised trials. Lancet. 2011;377:1760-9.

36. Landells LJ, Naidoo B, Robertson J, Clark P. NICE guidance on pirfenidone for treatingidiopathic pulmonary fibrosis. Lancet Respir Med. 2013;1:191-2.

37. King TE, Bradford WZ, Castro-Bernardini S et al; ASCEND Study Group. A phase 3trial of pirfenidone in patients with idiopathic pulmonary fibrosis. N Engl J Med. 2014;370:2083-92.

38. Karimi-Shah BA, Chowdhury BA. Forced vital capacity in idiopathic pulmonary fibrosis-FDA review of pirfenidone and nintedanib. $\mathrm{N}$ Engl J Med. 2015; 372: 1189-91.

39. Noble PW, Albera C, Bradford WZ et al. Pirfenidone for idiopathic pulmonary fibrosis: analysis of pooled data from three multinational phase 3 trials. Eur Resp J. 2016;47:243-53. 
40. Nathan SD, Albera C, Bradford WZ et al. Effect of pirfenidone on mortality: pooledanalyses and meta-analyses of clinical trials in idiopathic pulmonary fibrosis. Lancet Respir Med. 2017;5:33-41.

41. Cottin V, Maher T. Long-term clinical and real-world experience with pirfenidone in the treatment of idiopathic pulmonary fibrosis. Eur Respir Rev. 2015;24:58-64

42. Ogura T, Azuma A, Inoue $\mathrm{Y}$ et al. All-case post-marketing surveillance of 1371 patients treated with pirfenidone for idiopathic pulmonary fibrosis. Respir Investig. 2015;53:232-41.

43. Lancaster L, Albera C, Bradford WZ et al. Safety of pirfenidone in patients with idiopathic pulmonary fibrosis: integrated analysis of cumulative data from 5 clinical trials. BMJ Open Respir Res. 2016;3:e000105.

44. Nathan SD, Albera C, Bradford WZ et al. Effect of continued treatment with pirfenidone following clinically meaningful declines in forced vital capacity: analysis of data fromthree phase 3 trials in patients with idiopathic pulmonary fibrosis. Thorax. 2016;71:429-35.

45. Loeh B, Drakopanagiotakis F, Bandelli GP et al. Intraindividual Response to Treatment with Pirfenidone in Idiopathic Pulmonary Fibrosis. Am J Respir Crit Care Med. 2015;191:110-3.

46. Richeldi L, Costabel U, Selman M et al. Efficacy of a tyrosine kinase inhibitor in idiopathic pulmonary fibrosis. N Engl J Med. 2011;365:1079-87.

47. Richeldi L, du Bois RM, Raghu G et al; INPULSIS Trial Investigators. Efficacy and Safety of Nintedanib in Idiopathic Pulmonary Fibrosis. N Engl J Med. 2014;370:2071-82.

48. Costabel U, Inoue Y, Richeldi L et al. Efficacy of Nintedanib in Idiopathic Pulmonary Fibrosis across Prespecified Subgroups in INPULSIS. Am J Respir Crit Care Med. 2015;193:178-85.

49. Raghu G, Wells AU, Nicholson AG et al. Effect of Nintedanib in Subgroups of Idiopathic Pulmonary Fibrosis by Diagnostic Criteria. Am J Respir Crit Care Med. 2017;195:78-85

50. Corte T, Bonella F, Crestani B et al. Safety, tolerability and appropriate use of nintedanib in idiopathic pulmonary fibrosis. Respir Res. 2015;16:116.

51. Loveman E, Copley VR, Scott DA, Colquitt JL, Clegg AJ, O'Reilly KM. Comparing new treatments for idiopathic pulmonary fibrosis - a network meta-analysis. BMC Pulm Med. 2015;15:37.

52. Canestaro WJ, Forrester SH, Raghu G, Ho L, Devine BE. Drug Treatment of IdiopathicPulmonary Fibrosis Systematic Review and Network MetaAnalysis. Chest. 2016;149:756-66.

53. Trawinska MA, Rupesinghe RD, Hart SP. Patient considerations and drug selection in the treatment of idiopathic pulmonary fibrosis. Ther Clin Risk Manag. 2016;12:563-74.

54. King CS, Nathan SD. POINT: Should All Patients With Idiopathic Pulmonary Fibrosis, Even Those With More Than Moderate Impairment, Be Treated with Nintedanib or Pirfenidone? Yes. Chest. 2016;150:273-5.

55. Kolb M, Richeldi L, Behr J et al. Nintedanib in patients with idiopathic pulmonary fibrosis and preserved lung volume. Thorax. 2016;0:1-7.

56. Wuyts WA, Kolb M, Stowasser S, Stansen W, Huggins JT, Raghu G. First Data on Efficacy and Safety of Nintedanib in Patients with Idiopathic Pulmonary Fibrosis and Forced Vital Capacity of $\leq 50 \%$ of Predicted Value. Lung. 2016;194:739-43.

57. Sakamoto S, Itoh T, Muramatsu Y et al. Efficacy of pirfenidone in patients with advanced-stage idiopathic pulmonary fibrosis. Intern Med. 2013;52: 2495-2501.

58. Wells AU. Combination therapy in idiopathic pulmonary fibrosis: the way ahead will be hard. Eur Respir J. 2015;45:1208-10.

59. Wuyts WA, Antoniou KM, Borensztajn K et al. Combination therapy: the future of management for idiopathic pulmonary fibrosis? Lancet Respir Med. 2014;2:933-42.
60. King TE Jr., Albera C, Bradford WZ et al. All-Cause Mortality Rate in Patients withIdiopathic Pulmonary Fibrosis. Implications for the Design and Execution of ClinicalTrials. Am J Respir Crit Care Med. 2014;189:825-31.

61. Ogura T, Taniguchi H, Azuma A et al. Safety and pharmacokinetics of nintedanib andpirfenidone in idiopathic pulmonary fibrosis. Eur Respir J. 2015;45:1382-92.

62. Murray LA, Argentieri RL, Farrell FX et al. Hyper-responsiveness of IPF/ UIP fibroblasts: interplay between TGFbeta1, IL-13 and CCL2. Int J Biochem Cell Biol. 2008;40:2174-82.

63. Wang $Q$, Usinger $\mathrm{W}$, Nichols $B$ et al. Cooperative interaction of CTGF and TGF-beta in animal models of fibrotic disease. Fibrogenesis Tissue Repair. 2011;4:4

64. Raghu G, Scholand MB, de Andrade Joao et al. FG-3019 anti-connective tissue growth factor monoclonal antibody: results of an open-label clinical trial in idiopathic pulmonary fibrosis. Eur Respir J. 2016;47:1481-91.

65. Wynn TA. Fibrotic disease and the $\mathrm{T}(\mathrm{H}) 1 / \mathrm{T}(\mathrm{H}) 2$ paradigm. Nat Rev Immunol. 2004;4:583-94.

66. Hancock A, Armstrong L, Gama R, Millar A. Production of interleukin 13 by alveolar macrophages from normal and fibrotic lung. Am J Respir Cell Mol Biol. 1998;18:60-5.

67. Blobe GC, Schiemann WP, Lodish HF. Role of Transforming Growth Factor $\beta$ in Human Disease. N Engl J Med. 2000;342:1350-8.

68. Wipff PJ, Hinz B. Integrins and the activation of latent transforming growth factor beta1 - an intimate relationship. Eur J Cell Biol. 2008;87:601-15.

69. O'Dwyer DN, Dickson RP, Moore BB. The Lung Microbiome, Immunity, and the Pathogenesis of Chronic Lung Disease. J Immunol. 2016;196:4839-47.

70. Han MK, Zhou Y, Murray S et al. Association Between Lung Microbiome and Disease Progression in IPF: A Prospective Cohort Study. Lancet Respir Med. 2014;2:548-56.

71. Huang Y, Ma SF, Espindola MS et al. Microbes associate with host innate immune response in idiopathic pulmonary fibrosis. Am J Respir Crit Care Med (in press).

72. Molyneaux PL, Cox MJ, Willis-Owen SA et al. The role of bacteria in the pathogenesis and progression of idiopathic pulmonary fibrosis. Am J Respir Crit Care Med. 2014;190:906-13.

73. Molyneaux PL, Willis-Owen SA, Cox MJ et al. Host-Microbial interactions in Idiopathic Pulmonary Fibrosis. Am J Respir Crit Care Med (in press).

74. Shulgina L, Cahn AP, Chilvers ER et al. Treating idiopathic pulmonary fibrosis with the addition of co-trimoxazole: a randomised controlled trial Thorax. 2013;68:155-62.

75. Toonkel RL, Hare JM, Matthay MA, Glassberg MK. Mesenchymal Stem Cells and Idiopathic Pulmonary Fibrosis. Am J Respir Crit Care Med. 2013;188133-.40.

76. Glassberg MK, Minkiewicz J, Toonkel RL et al. Allogeneic human mesenchymal stem cells in patients with idiopathic pulmonary fibrosis via intravenous delivery (AETHER): a phase I, safety, clinical trial. Chest (in press).

77. Brownell R, Kaminski N, Woodruff PG et al. Precision Medicine: The New Frontier in Idiopathic Pulmonary Fibrosis. Am J Respir Crit Care Med. 2016;193:1213-18.

78. Clarke DL, Murray LA, Crestani B, Sleeman MA. Is personalised medicine the key to heterogeneity in idiopathic pulmonary fibrosis? Pharmacol Ther 2017;169:35-46.

79. Collins FS, Varmus H. A New Initiative on Precision Medicine. N Engl Med. 2015; 372:793-795.

80. Raghu G, Brown KK, Collard HR et al. Efficacy of simtuzumab versus placebo in patients with idiopathic pulmonary fibrosis: a randomised, double-blind, controlled, phase 2 trial. Lancet Respir Med. 2017;5:22-32. 